\title{
The ubiquitin conjugating enzyme UBE2L3 regulates TNF $\alpha$-induced linear ubiquitination
}

Cell Research (2014) 24:376-379. doi:10.1038/cr.2013.133; published online 24 September 2013

\section{Dear Editor,}

The inducible and reversible modification of proteins with polyubiquitin chains has emerged as one of the most common and versatile means of protein modification. Ubiquitin contains 7 lysine residues, each of which can serve as an acceptor site for formation of polyubiquitin chains. In addition, the C-terminal glycine of one ubiquitin molecule can form a conventional peptide bond with the N-terminal methionine of an acceptor ubiquitin, leading to the formation of linear polyubiquitin chains. Linear ubiquitin chains stabilize proteins and play critical roles in signaling complex assembly. The linear ubiquitin chain assembly complex (LUBAC) consists of at least 3 proteins: HOIP (also known as RNF31), HOIL-1L (also known as RBCK1), and SHARPIN [1-6]. LUBAC is the only E3 ligase known to assemble linear polyubiquitin chains. Importantly, LUBAC was shown to target components of the canonical NF- $\kappa \mathrm{B}$ signaling pathway, which is vital for development of inflammation and immunity $[2,5]$. Our understanding of linear ubiquitination primarily comes from studies involving $\mathrm{TNF} \alpha$ signaling. Upon stimulation with the cytokine TNF $\alpha$, LUBAC is recruited to the TNF $\alpha$ receptor 1 (TNFR1) $[1,5]$. LUBAC regulates $\mathrm{TNF} \alpha$ signaling by targeting the regulatory protein, NF- $\kappa B$ essential modulator (NEMO), for nondegradative linear ubiquitination. Depletion of LUBAC components by RNA interference or genetic ablation attenuates cytokine-driven NF- $\mathrm{KB}$ signaling $[1-5,7]$.

LUBAC is a $600 \mathrm{kDa}$ multimeric complex $[6,8,9]$. However, the stoichiometry and additional components associated with the LUBAC protein complex remain unknown. We used affinity protein purification in HEK293 cells stably expressing Flag-tagged HOIL-1L, HOIP and SHARPIN, followed by mass spectrometry to identify proteins that interact with LUBAC and contribute to its function. For data processing, we compared the affinity purification coupled with mass spectrometry (AP-MS) data of LUBAC with our laboratory database containing 208 protein complexes from HEK293 cells. Our database analysis tool, termed ZSPORE, is designed to identify high confidence interacting proteins (HCIPs) and filter out nonspecific binding proteins (NSBP) from AP-MS datasets [10]. ZSPORE integrates three parameters: z-score based on total spectral counts (z-score $>$ 5; $P<0.0001)$, prey occurrence $(<5 \%$, as a measure of uniqueness) and reproducibility ( $\geq 50 \%$ in 4 runs). In total, 32 HCIPs were uncovered (Supplementary information, Table S1). Reciprocal interactions among HOIL$1 \mathrm{~L}$, HOIP and SHARPIN were detected, indicating the strong associations among these three LUBAC components. In addition, HOIL-1L, HOIP and SHARPIN formed individual subnetworks with unique partners. Several known interactors, such as TRAF2, BIRC2 (also known as cIAP1), FAM105B (also termed OTULIN) and DZIP3, were present in the LUBAC interactome (Supplementary information, Figure S1A). However, $70 \%$ of the interactions were not found in literature searches or the STRING and BioGRID databases (Supplementary information, Figure S1A). Gene Ontology (GO) term enrichment analysis indicates that $41 \%$ of HCIPs are involved in the ubiquitination process, including several E3 ligases, deubiquitinases and one ubiquitin conjugating E2 enzyme (UBE2L3, also known as UbcH7 or UbcM4) (Supplementary information, Figure S1A). To determine whether these protein associations were dependent on $\mathrm{TNF} \alpha$ stimulation, we compared the ratio of total spectral counts with or without $\mathrm{TNF} \alpha$ treatment. Under the experimental conditions tested, TNF $\alpha$ treatment did not induce $>5$ fold changes in normalized spectral counts, suggesting that the LUBAC network represents a relatively homeostatic interactome.

Co-immunoprecipitation (co-IP) in HEK293 cells was used to confirm some interactions; 12 of the 13 interactions examined were validated by co-IP (Supplementary information, Figure S1A). The results identify potential LUBAC-associated proteins although the HCIP may also define binding partners involved in LUBAC-independent functions. For functional screening 10 HCIPs were transfected into HEK293 cells along with a NF- $\mathrm{KB}$ reporter and Renilla control. Ectopic expression of the ubiquitin conjugating E2 enzyme UBE2L3 activates NF- $\kappa B$ re- 
A

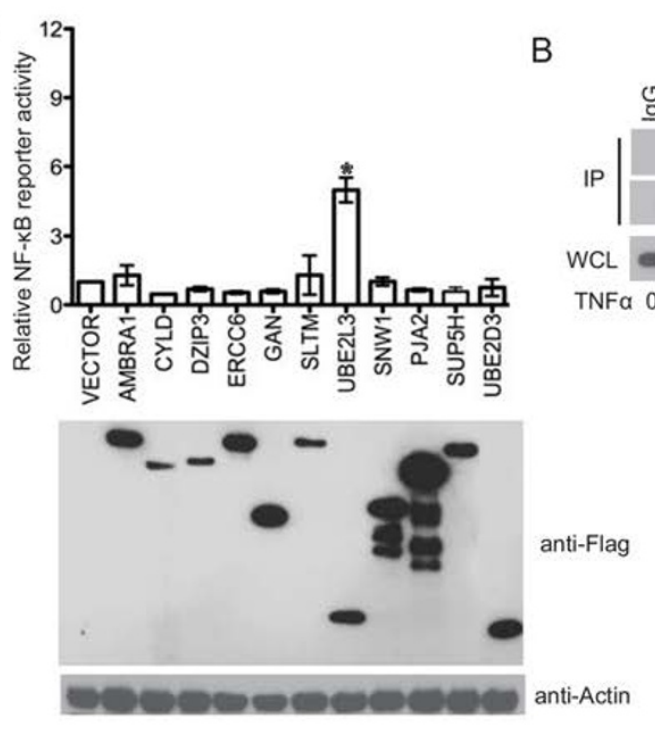

D

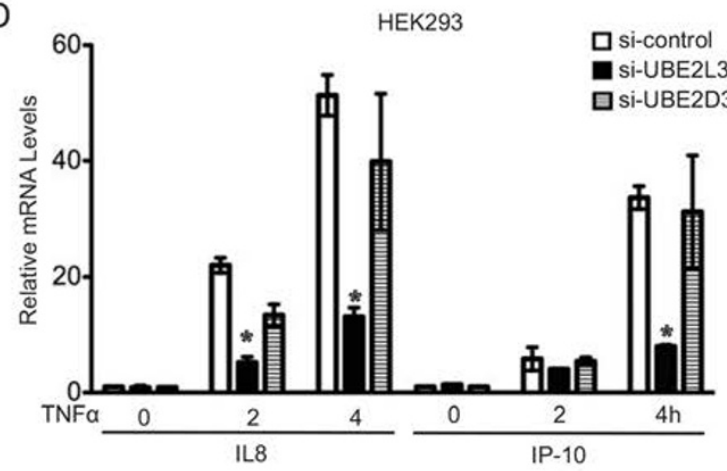

$\mathrm{F}$

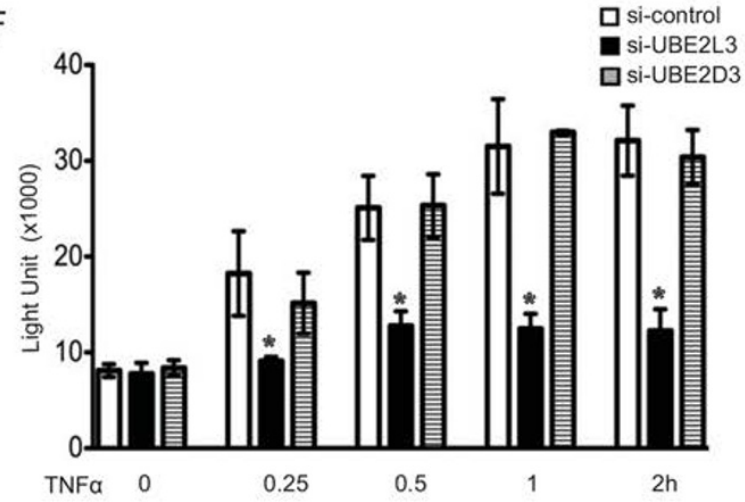

C

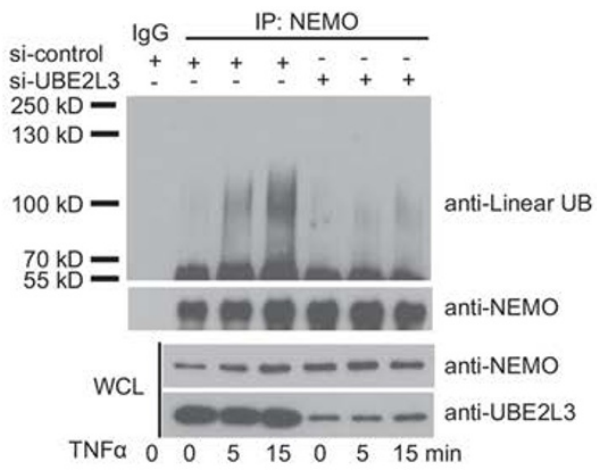

E
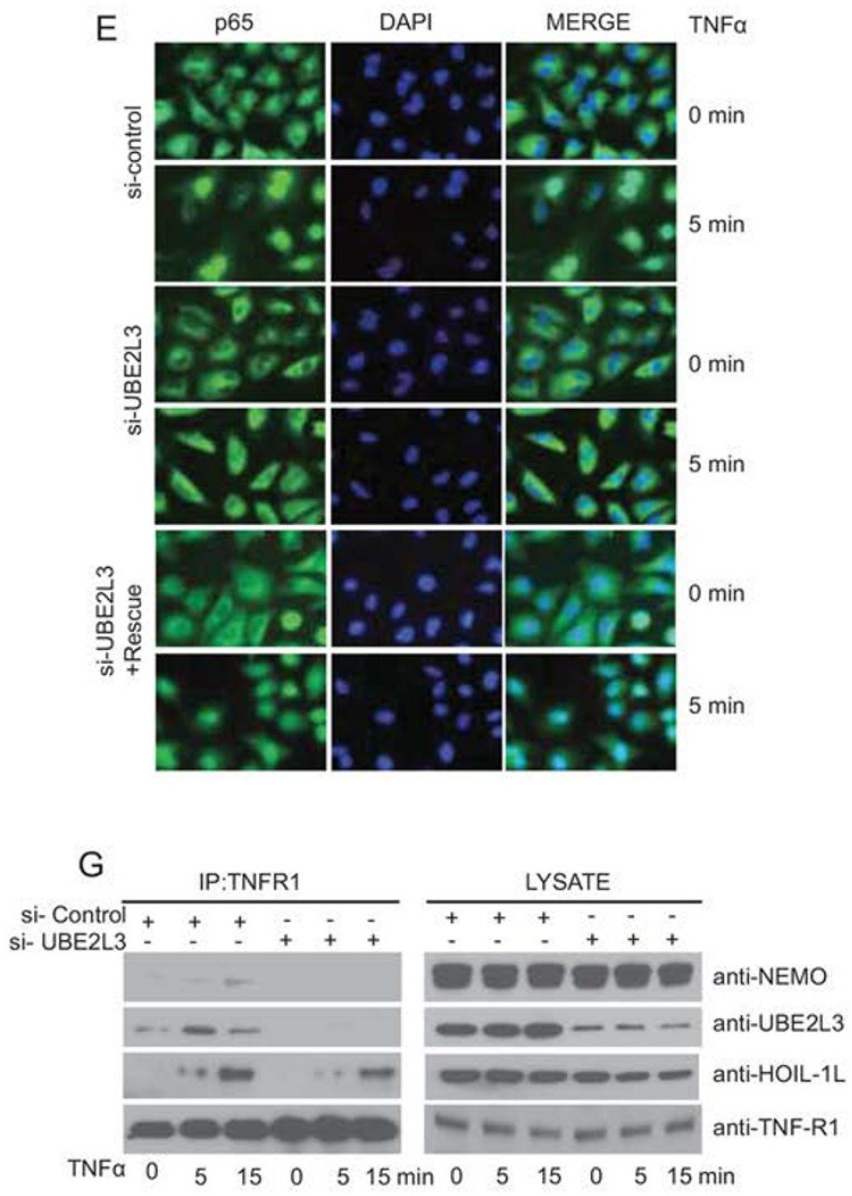

dogenous HOIL-1L interacts with endogenous UBE2L3 in vivo and these associations are stable following TNF $\alpha$ stimulation (Figure 1B). Direct interactions between UBE2L3 and HOIL-1L were also confirmed using bacterially expressed UBE2L3 and LUBAC proteins (Supplementary information, Figure S1C).

Using an antibody specific for linear ubiquitin chains [11], we examined the ability of UBE2L3 to support 


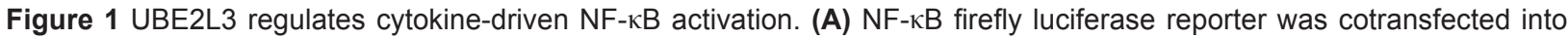
HEK293 cells with control Renilla luciferase and 10 LUBAC HCIPs or UBE2D3. Luciferase activity was measured after $48 \mathrm{~h}$. Data are pooled from 3 experiments. Standard deviation bars are shown. ${ }^{*} P<0.001$. Western blot shows expression levels from one representative experiment. (B) HEK293 cells were treated with $10 \mathrm{ng} / \mathrm{ml}$ TNF $\alpha$ for the indicated times before cell lysis. Immunoprecipitation with anti-HOIL-1L antibody was followed by probing blot with the indicated antibldies. Whole cell lysates (WCL) were used as controls to compare input levels. (C) HEK293 cells were treated with control or UBE2L3 siRNA, after $48 \mathrm{~h}$ cells were stimulated with $10 \mathrm{ng} / \mathrm{ml}$ TNF $\alpha$ for the indicated period. Cell lysates were immunoprecipitated with anti-NEMO antibody and blotted as indicated. (D) HEK293 cells were transfected with scrambled siRNA control, UBE2L3 siRNA or UBE2D3 siRNA. After $48 \mathrm{~h}$ the cells were stimulated with $10 \mathrm{ng} / \mathrm{ml}$ TNF $\alpha$ for 2 or $4 \mathrm{~h}$. Relative mRNA levels for the indicated NF-kB-dependent genes were determined by qPCR. Standard deviation bars are depicted. ${ }^{*} P<0.001$. (E) After transfection with si-UBE2L3, si-control or si-UBE2L3 plus a rescue UBE2L3 cDNA, HeLa cells were stimulated with 10 ng/ $\mathrm{ml} \mathrm{TNF} \alpha$ for 0 or $5 \mathrm{~min}$. Cells were stained for detection of p65 and nuclei (DAPI). (F) HEK293 cells were transfected with siRNA control, UBE2L3 siRNA, or UBE2D3 siRNA. After $48 \mathrm{~h}$ the cells were stimulated with $10 \mathrm{ng} / \mathrm{ml}$ TNF $\alpha$ for the indicated times. Binding of nuclear p65 to consensus NF-kB DNA elements was assayed with anti-p65 antibody, developed with a secondary HRP-conjugated anti-IgG antibody and measured by luminescence. Standard deviation bars are depicted. ${ }^{*} P$ $<0.001$. (G) HEK293 cells were treated with si-control or si-UBE2L3. After $48 \mathrm{~h}$ cells were stimulated with TNF $\alpha$ for the indicated times. Cell lysates were immunoprecipitated with anti-TNFR1antibodies and probed with the indicated antibodies.

linear ubiquitin synthesis. Consistent with previous reports [6], UBE2L3 cooperates with LUBAC to support in vitro formation of linear ubiquitin chains. The absence of UBE2L3 or substitution of a catalytically inactive UBE2L3 containing a mutated active site cysteine (C86), failed to generate linear ubiquitin (Supplementary information, Figure S2A). UBE2L3 also supports linear ubiquitination of NEMO in vitro (Supplementary information, Figure S2B). To determine wether UBE2L3 facilitates conjugation of linear ubiquitin chains in cells, wild-type or mutant UBE2L3 was co-transfected with NEMO and LUBAC components. Linear ubiquitin was detected in NEMO immunoprecipitates when wild-type UBE2L3 was transfected along with HOIP plus HOIL1L and/or SHARPIN (Supplementary information, Figure S2C). However, linearly ubiquitinated NEMO was not observed when cells were co-transfected without multiple LUBAC components or with catalytically inactive UBE2L3 (Supplementary information, Figure S2C). To complement the results obtained by UBE2L3 overexpression, we silenced UBE2L3 with siRNA. Cells were stimulated with TNF $\alpha$ prior to immunoprecipitation of NEMO. In control cells TNF $\alpha$ treatment increases linear ubiquitination of endogenous NEMO, but knockdown of UBE2L3 decreases TNF $\alpha$-induced NEMO linear ubiquitination (Figure 1C). These experiments indicate that UBE2L3 plays an important role in the assembly of NEMO linear polyubiquitin chains in vitro and in vivo.

As linear ubiquitination is pivotal for cytokine-mediated activation of NF-kB signaling, we speculated that UBE2L3 could regulate TNF $\alpha$-driven NF- $\kappa B$ signaling. To test this hypothesis, we first examined the effect of UBE2L3 knockdown on TNF $\alpha$-induced gene expression. Depletion of UBE2L3 by siRNA inhibited TNF $\alpha$-induced
IL8 and IP-10 gene expression in HEK293 (Figure 1D), and IL6, IL8 and IP-10 expression in HeLa cells (Supplementary information, Figure S3A). A UBE2L3 rescue construct validated siRNA specificity (Supplementary information, Figure S3A). Notably, silencing of a different E2 (UBE2D3) did not significantly impact cytokine expression (Figure 1D and Supplementary information, Figure S3B). Furthermore, TNF $\alpha$-driven p65 nuclear translocation is also impaired after UBE2L3 depletion in HeLa cells, but is restored with a rescue UBE2L3 construct (Figure 1E and Supplementary information, Figure S3C). Similar results were obtained with HEK293 cells (Supplementary information, Figure S3D). Furthermore, treatment with UBE2L3 siRNA inhibits binding of the nuclear p65 NF- $\mathrm{KB}$ transcription factor to NF- $\mathrm{KB}$ consensus sequences (Figure 1F). Consistently, knockdown of UBE2D3 has little effect on nuclear p65 NF$\kappa \mathrm{B}$ binding activity (Figure $1 \mathrm{~F}$ ). In addition, silencing UBE2L3 impairs TNF $\alpha$-induced I $\kappa \mathrm{B} \alpha$ phosphorylation and I $\mathrm{I} B \alpha$ recovery, but the presence of UBE2L3 siRNA has little impact on cytokine-induced JNK phosphorylation (Supplementary information, Figure S3E). The latter results parallel findings with HOIL-1-deficient MEFs [2]. The transient pulse of $I \kappa B \alpha$ phosphorylation following UBE2L3 knockdown and TNF $\alpha$ treatment was paralleled by a brief pulse of phosphorylated p65 (Supplementary information, Figure S3E). These results imply that NF$\kappa \mathrm{B}$ signaling is initiated while UBE2L3 is silenced, however signal propagation is not sustained without UBE2L3 (Supplementary information, Figure S3E).

$\mathrm{TNF} \alpha$ triggers a complex signaling mechanism that requires assembly of multiple proteins on the cytosolic face of the ligated TNFR1 [1, 5]. HEK293 cells were treated with siRNA and then stimulated with TNF $\alpha$ for 
various times before cell lysis. Cell lysates were immunoprecipitated with anti-TNFR1 and probed to detect endogenous proteins associated with TNFR1. TNF $\alpha$ treatment enhances UBE2L3 and HOIL-1L interaction with the TNFR1 receptor complex (Figure 1G). The level of NEMO associated with TNFR1 also increases after ligand treatment. However, silencing UBE2L3 blocks the association of TNFR1 with NEMO, while HOIL-1L is still recruited to the receptor complex (Figure 1G). Taken together, these results demonstrate that recruitment of NEMO to TNFR1 is UBE2L3 dependent.

In vitro LUBAC can generate linear ubiquitin chains with multiple E2s including UBE2L3 and UBE2D3 [2, $4-6,8,9]$. However, the role of these E2s in cells was not investigated. We find that UBE2D3 fails to associate with LUBAC by co-IP. It also lacks the capacity to activate NF-қВ reporter. Moreover, UBE2D3 depletion failed to inhibit p65 binding to DNA and did not modulate cytokine transcription. Conversely, we identify UBE2L3 as a cognate E2 for LUBAC based on multiple lines of evidence: (1) proteomics identified the association between HOIL-1L and UBE2L3, which was further validated by endogenous protein co-IP; (2) UBE2L3 regulates TNF $\alpha$ stimulated linear ubiquitination of NEMO in vivo; and (3) silencing UBE2L3 blocks NEMO recruitment to TNFR1, TNF $\alpha$-induced I $\mathrm{B} \mathrm{B} \alpha$ and p65 phosphorylation, transcription factor binding to NF- $\kappa \mathrm{B}$ promoter elements, p65 translocation, and proinflammatory gene expression. Thus, UBE2L3 is the predominant E2 for LUBACdependent NF- $\mathrm{kB}$ activity in vivo, although we cannot exclude minor roles of other E2s nor the possibility that UBE2L3 also mediates linear ubiquitination of other targets.

We propose a mechanistic model in which UBE2L3 associates with HOIL-1L in the LUBAC complex. When the LUBAC complex is recruited to TNFR1, UBE2L3 travels along and facilitates linear ubiquitination of NEMO, which is in turn recruited to TNF signaling complexes. NEMO contains two ubiquitin-binding sites that may crosslink and stabilize other ubiquitinated components associated with the activated TNFR1 complex. NEMO also functions as an adaptor protein for the IKK $\alpha / \mathrm{IKK} \beta$ kinases, which are recruited to and activated in TNFR1 complexes. The IKKs target I $\mathrm{I} B \alpha$ for phosphorylation and sustain NF- $\kappa \mathrm{B}$ signaling. Collectively, these results suggest that UBE2L3 plays a critical role in assembly, maintenance and coordination of the crowded TNFR1 signaling platform.

Genome-wide association studies using multiple independent cohorts indicate that genetic variants in the UBE2L3 region are associated with autoimmunity including systemic lupus erythematosus [12], rheumatoid arthritis [13] and Crohn's disease [14]. Furthermore, expression studies indicate that these risk haplotypes correlate with enhanced UBE2L3 RNA and protein expression $[12,14]$. As increased UBE2L3 expression enhances $\mathrm{NF}-\kappa \mathrm{B}$ activation, and increased levels of NF- $\kappa \mathrm{B}$ activity are linked to inflammatory and autoimmune diseases, the data suggest a mechanism by which UBE2L3 can influence pathogenesis of these disease.

\section{Acknowledgments}

We thank Dr Robert F Kelley and Genentech for generously supplying the anti-linear ubiquitin antibody. SL was funded by the John and Virginia Kaneb Immunology Fellowship. This study was supported by NIH grants AI089829 and AI099860.

\author{
Bishi $\mathrm{Fu}^{1, *}$, Shitao $\mathrm{Li}^{1,{ }^{1,}}$, Lingyan Wang ${ }^{1}$, \\ Michael A Berman ${ }^{1}$, Martin E Dorf ${ }^{1}$ \\ ${ }^{\prime}$ Division of Immunology, Department of Microbiology and Immunobiol- \\ ogy, Harvard Medical School, Boston, MA 02115, USA \\ *These two authors contributed equally to this work. \\ Correspondence: Martin E Dorf \\ E-mail: dorf@hms.harvard.edu
}

\section{References}

1 Haas TL, Emmerich CH, Gerlach B, et al. Mol Cell 2009; 36:831-844.

2 Tokunaga F, Sakata S, Saeki Y, et al. Nat Cell Biol 2009; 11:123-132.

3 Tokunaga F, Nakagawa T, Nakahara M, et al. Nature 2011; 471:633636.

4 Ikeda F, Deribe YL, Skanland SS, et al. Nature 2011; 471:637-641.

5 Gerlach B, Cordier SM, Schmukle AC, et al. Nature 2011; 471:591596.

6 Kirisako T, Kamei K, Murata S, et al. EMBO J 2006; 25:4877-4887.

7 Boisson B, Laplantine E, Prando C, et al. Nat Immunol 2012; 13:11781186.

8 Smit JJ, Monteferrario D, Noordermeer SM, et al. EMBO J 2012; 31:3833-3844.

9 Stieglitz B, Morris-Davies AC, Koliopoulos MG, et al. EMBO Rep 2012; 13:840-846.

10 Li S, Dorf ME. J Proteomics Genomics Res 2013; 1:9-19.

11 Matsumoto ML, Dong KC, Yu C, et al. J Mol Biol 2012; 418:134-144.

12 Wang S, Adrianto I, Wiley GB, et al. Genes Immun 2012; 13:380-387.

13 Orozco G, Eyre S, Hinks A, et al. Ann Rheum Dis 2011; 70:463-468.

14 Fransen K, Visschedijk MC, van Sommeren S, et al. Hum Mol Genet 2010; 19:3482-3488

(Supplementary information is linked to the online version of the paper on the Cell Research website.) 